\title{
Optical characterization of subwavelength-scale solid immersion lenses
}

\author{
Myun-Sik Kim*a ${ }^{* a}$ Toralf Scharf ${ }^{\mathrm{a}}$, Mohammad Tahdiul Haq ${ }^{\mathrm{b}}$, Wataru Nakagawa ${ }^{\mathrm{b}}$, \\ and Hans Peter Herzig ${ }^{a}$ \\ ${ }^{a}$ Optics \& Photonics Technology Laboratory, Ecole Polytechnique Fédérale de Lausanne (EPFL), \\ Neuchâtel, CH-2000, Switzerland \\ ${ }^{\mathrm{b}}$ Department of Electrical and Computer Engineering, Montana State University, P.O. Box 173780, \\ Bozeman, Montana 59717-3780, USA
}

\begin{abstract}
We present the fabrication and optical characterization of nano-scale solid immersion lenses (nano-SILs) with sizes down to a subwavelength range. Submicron-scale cylinders fabricated by electron-beam lithography (EBL) are thermally reflowed to form a spherical shape. Subsequent soft lithography leads to nano-SILs on transparent substrates, i.e. glass, for optical characterization with visible light. The optical characterization is performed using a high-resolution interference microscope (HRIM) with illumination at $642 \mathrm{~nm}$ wavelength. The measurements of the 3D amplitude and phase fields provide information on the spot size and the peak intensity. In particular, the phase measurement is a more convincing proof of the Airy disc size reduction rather than the full-width at half maximum (FWHM) spot size. The focal spots produced by the nano-SILs show both spot-size reduction and enhanced optical intensity, which are consistent with the immersion effect. In this way, we experimentally confirm the immersion effect of a subwavelength-size SIL ( $d=530$ $\mathrm{nm}$ and $\mathrm{h}=45 \mathrm{~nm}$ ) with a spot reduction ratio of 1.35 , which is less than the expected value of 1.5 , most likely due to the slightly non-ideal shape of the nano-SIL.
\end{abstract}

Keywords: electron-beam lithography, thermal reflow, soft lithography, solid immersion lens (SIL), nano-SIL, subwavelength-size SIL, spot size reduction, 3D amplitude and phase measurement

\section{INTRODUCTION}

In 1678, Robert Hooke first discussed the technique of immersion to improve the imaging performance of a microscope in his book Microscopium ${ }^{1}$. Afterward, Sir David Brewster proposed the immersion of the objective in $1812^{2}$. The concept of homogeneous immersion and the immersion objective preceded Ernst Abbe's pioneering work, in which he constructed the first oil-immersion lens in developing the imaging theory of microscopy ${ }^{3}$. Abbe also developed the standard measure of performance of an objective lens, the numerical aperture (NA), which is defined as ${ }^{4}$

$$
N A=n \cdot \sin \theta,
$$

where $\theta$ is the angle a ray makes with the optical axis (i.e. half the angle of the focus cone), and $n$ is the refractive index of the medium through which the rays pass. Until S. Mansfield et al. developed a new immersion concept in 1990, which is termed solid immersion lens $(S I L)^{5}$, lens makers and researchers had focused on improving the performance of objectives and finding proper immersion liquids. The concept of solid immersion is derived from the idea that focusing in the center of a hemispherical solid leads to normal incidence of the rays with respect to the spherical interface. Therefore, there is no refraction at the interface between the spherical solid and the surrounding medium (e.g. air), leading to an increase in the NA by a factor of $n$ (the refractive index of the solid medium) as seen in Eq. (1). During the last two decades, numerous studies of SILs and their applications have been carried out. At first, SILs were limited to macroscopic size (i.e. millimeter scale) due to a lack of fabrication technologies. Advances in micro- and nanofabrication technologies enabled the development of different types of SILs, including diffractive SILs ${ }^{6}$, micrometer-size SILs ${ }^{7-10}$, nanoscale spherical lenses ${ }^{11}$, and wavelength-scale SILs ${ }^{12}$. In general, for structures smaller than the optical wavelength, design methods for larger devices, such as ray optics, are not applicable. More specifically, subwavelength-scale lenses cannot simply be considered to be refractive optical surfaces. However, recent modeling work has shown that subwavelength scale SILs are still expected to produce a reduced size focal $\operatorname{spot}^{12}$, the so-called immersion effect. Recently, we for the first time reported the experimental demonstration of the immersion effect in subwavelength-scale SILs ${ }^{13}$.

*myunsik.kim@epfl.ch; phone 41-32-718-3279; fax 41-32-718-3201; http://opt.epfl.ch/

Advanced Fabrication Technologies for Micro/Nano Optics and Photonics V, edited by Winston V. Schoenfeld,

Raymond C. Rumpf, Georg von Freymann, Proc. of SPIE Vol. 8249, 82491B - C 2012 SPIE

CCC code: $0277-786 X / 12 / \$ 18 \cdot$ doi: $10.1117 / 12.905472$

Proc. of SPIE Vol. 8249 82491B-1 
In this paper we present the details of the fabrication and optical characterization of nano-scale SILs. First, we discuss the design and the fabrication processes: (1) cylindrical patterns written by EBL, (2) thermal reflow, (3) replication by soft lithography, and (4) structural characterization of the size and surface profile. Second, optical characterization using the HRIM is explained in detail.

\section{FABRICATION}

Electron-beam lithography (EBL) has been used for nano-pattering and structuring. The EBL resist, polymethyl methacrylate (PMMA), has in general been considered as an etch mask for further fabrication steps rather than a host material for direct nanostructuring. Since PMMA is a transparent thermoplastic, the thermal reflow structuring ${ }^{14,15}$ can be applied and a final pattern can be directly used as an optical element in the visible spectrum. Recently, micro-size structuring by EBL and a following thermal reflow have been reported ${ }^{16}$. However, in order to achieve high-resolution nano-size patterns, one needs a very thin PMMA coating, which is typically below $500 \mathrm{~nm}$. To the best of our knowledge, there has been no previous detailed investigation of complete reflow structuring at these dimensions. In this section, we present the detailed fabrication processes of such nano-SILs.

\subsection{Design}

Figure 1 shows the schematic for the transformation from a cylinder to a spherical cap by thermal reflow above the glass transition temperature $\left(T_{g}\right)$ of a polymer. For simplicity, we assume three aspects: (1) the sidewall of cylindrical pattern is vertical, (2) no moving of the bottom edge, in other words, the radius of cylinder $r$ stays the same, and (3) no volume change during the reflow process, which means the volume of the cylinder $V_{c y l i n d e r}$ equals that of the spherical cap $V_{c a p}$.
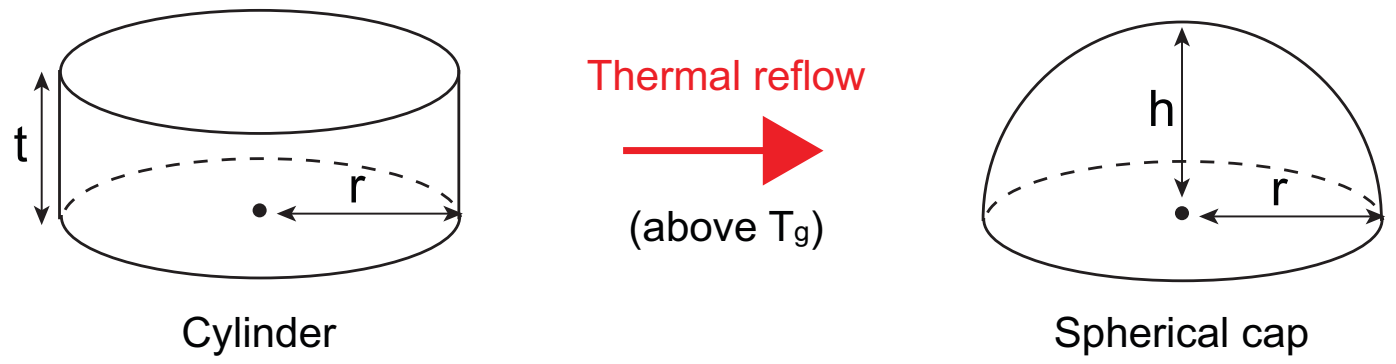

Figure 1. Schematic of the transformation from a cylinder to a spherical cap by thermal reflow. For simplicity, we make three assumptions: (1) the sidewall of the cylinder is vertical, (2) no moving of the bottom edge $[r=$ constant $]$, and (3) no volume change during the reflow process $\left[V_{c y l i n d e r}=V_{c a p}\right]$.

The volume of a cylinder and that of a spherical cap are written in Eqs. (2) and (3), respectively,

$$
\begin{aligned}
& V_{\text {cylinder }}=\pi r^{2} t, \\
& V_{\text {cap }}=\frac{1}{6} \pi h\left(3 r^{2}+h^{2}\right),
\end{aligned}
$$

where $r$ and $t$ are the radius and the thickness of the cylinder, respectively, and $h$ is the height of the spherical cap. In order to find design parameters to obtain the hemispherical shape, we set $r=h$ in Eqs. (2) and (3) and rewrite them as

$$
\begin{aligned}
& V_{\text {cylinder }}=\pi h^{2} t, \\
& V_{\text {cap }}=\frac{1}{6} \pi h\left(4 h^{2}\right) .
\end{aligned}
$$

Consequently, equating Eqs. (4) and (5) $\left[V_{c y l i n d e r}=V_{c a p}\right]$, the required thickness of the cylinder is given as

$$
t=\frac{2}{3} h \text {. }
$$


We design two sizes of the hemispherical (i.e. ideally shaped) nano-SIL, considering a wavelength of $642 \mathrm{~nm}$. One is at a size approximately equal to the wavelength, with diameter $d(=2 r) 600 \mathrm{~nm}(=\sim \lambda)$. The other is a subwavelength-size SIL, which has a diameter of $450 \mathrm{~nm}$. Equation (6) leads to the corresponding required thicknesses of the cylindrical patterns as $200 \mathrm{~nm}$ and $150 \mathrm{~nm}$, respectively.

\subsection{Electron-beam lithography}

The polymer nanostructures are fabricated using EBL on Silicon substrates with a thin PMMA resist layer (MicroChem Corp., 950k molecular weight). The Silicon substrates are used to improve sample conductivity and thereby reduce charging effects. Two sizes of PMMA cylinder, (1) $d=600 \mathrm{~nm}$ and $t=200 \mathrm{~nm}$ and (2) $d=450 \mathrm{~nm}$ and $t=150 \mathrm{~nm}$, are fabricated, as shown in the scanning electron microscope (SEM) images of Figs. 2(a) and 2(b), respectively. An area of approximately $4 \mu \mathrm{m}$ square has been exposed to pattern a cylinder in the center. Since PMMA is a positive resist, during the development process this area is removed, leaving a free-standing cylinder of PMMA.
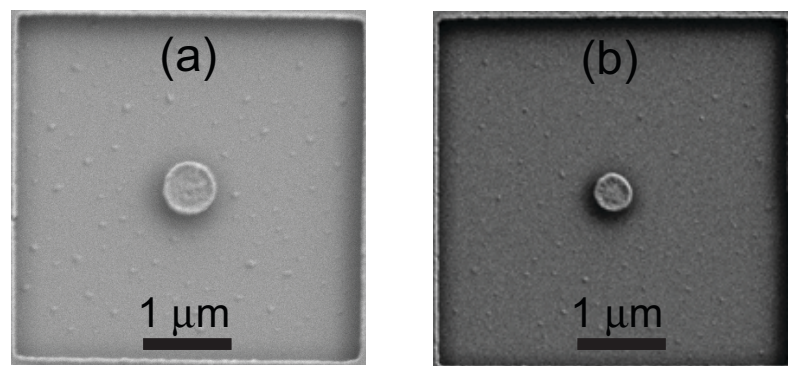

Figure 2. SEM images of PMMA cylindrical structures on the silicon substrate: (a) diameter $=600 \mathrm{~nm}$ and height $=200$ $\mathrm{nm}$ and (b) diameter $=450 \mathrm{~nm}$ and height $=150 \mathrm{~nm}$.

\subsection{Thermal reflow}

In general, the reference temperature for thermal reflow is the glass transition temperature of polymers, which is approximately between $110^{\circ} \mathrm{C}$ and $120^{\circ} \mathrm{C}$ for PMMA. Note that the $T_{g}$ of PMMA varies depending on the molecular weight $\mathrm{M}_{\mathrm{w}}$, which also varies depending on the exposure dose of the electron beam ${ }^{16}$. Several studies report that reflow below $120^{\circ} \mathrm{C}$ led only to smoothing, the reduction of surface roughness, partial reflow, or partial deformation of the structures $^{16-18}$. Therefore, we set our reference temperature to be $120^{\circ} \mathrm{C}$.

Among various conditions, we obtained optimal spherical structures when the 600 -nm diameter cylinders were reflowed at $150^{\circ} \mathrm{C}$ for 20 minutes on a hot plate, and the 450 -nm cylinders at $140^{\circ}$ for 20 minutes.

\subsection{Replication on a transparent substrate}

Since silicon is opaque in the visible spectrum, the reflowed spherical structures must be replicated on transparent substrates for optical characterization in transmission. To do so, we employ soft lighography, which is a well-established and proven method for the replication of nano-scale structures ${ }^{19}$. A UV-curable polymer (Norland, NOA 65, $\mathrm{n}=1.52$ ) is used to fill the replication mold, which is made of PDMS. The final spherical structures are molded on borosilicate glass cover slips (thickness $=150 \mu \mathrm{m}, \mathrm{n}=1.52$ ). Figures 3(a) and 3(b) show SEM images of the replicated spherical structures on glass substrates.
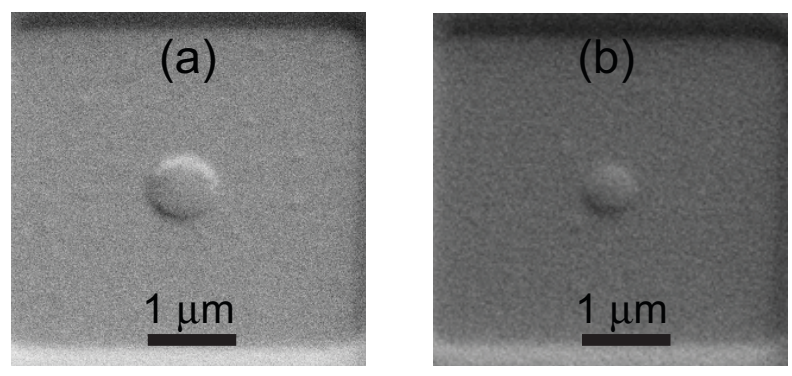

Figure 3. SEM images of polymer spherical structures replicated on the glass substrate after reflow: (a) diameter $=700$ $\mathrm{nm}$ and height $=54 \mathrm{~nm}$ and $(\mathrm{b})$ diameter $=530 \mathrm{~nm}$ and height $=45 \mathrm{~nm}$. 


\subsection{Structural characterization}

The SEM images provide an estimate of the variation of the diameter or the radius of the SIL (after reflow) with respect to the size of the cylinder (before reflow). Afterward, inspection using atomic force microscopy (AFM) leads to the surface profile of the SIL, which reveals the diameter and height of the spherical cap and the surface shape. The 600-nm cylinder was transformed into a spherical cap with a diameter of $700 \mathrm{~nm}$ and a height of $54 \mathrm{~nm}$, which yields a radius of curvature (ROC) of $1.16 \mu \mathrm{m}$, as shown Fig. 3(a). Figure 3(b) shows the 530-nm diameter and 45-nm height spherical cap $(\mathrm{ROC}=781 \mathrm{~nm})$ from the $450-\mathrm{nm}$ cylinder. Note that during the fabrication process, the volume change and the increase of the diameter led to a decrease in the height, which leads to a subhemispherical SIL $^{12}$. Moreover, in reality the sidewalls are not perfectly vertical, and this introduces some uncertainty in the optimum thickness of the cylinder. Currently, the thickness of the cylinder and different reflow conditions are under investigation in order to achieve the ideal hemispherical structure.

\section{OPTICAL CHARACTERIZATION}

For optical characterization we employ a high-resolution interference microscope, which is a proven tool to characterize micro- and nano-optical elements ${ }^{13,20-22}$ [details of the experiment are reported in the references]. The HRIM records the three-dimensional (3D) field distributions of highly confined light fields of NA $\geq 0.9$. The amplitude distributions reveal the FWHM size of focused spots, and the intensity profiles show the peak intensity enhancement when a tighter confinement is realized due to the immersion effect. The phase distributions are of interest because one can measure the exact size of the Airy disc, which represents the full spot size rather than the FWHM size. As measurements of the amplitude fields are subject to the diffraction limit of the observing objective, we use a 100X / NA1.4 immersion objective (Leica Microsystems, HXC PL APO) in order to obtain the highest resolution in oil immersion. The HRIM is equipped with a single-mode polarized laser of 642-nm wavelength (CrystaLaser, DL640-050-3).

Figure 4 shows the experimental geometries and the CCD camera images of the bottom plane of the 700-nm SIL with the focal spot, in which the dark point in the center is the SIL and the square is the 4- $\mu \mathrm{m}$ square area where the PMMA has been patterned. The focusing lens is a dry objective 50X / NA0.9 HXC PL APO (Leica Microsystems). In order to verify the immersion effect, we illuminate the sample with a focused beam at three different positions, as schematically illustrated in Fig. 4. First, we focus at the interface between the air and the glass substrate in the exposed region where there is no polymer, as shown Fig. 4(a). This represents the reference case. Second, we focus on the polymer-glass interface, illuminating through the 200-nm thick planar region of the polymer, as shown in Fig. 4(b). Comparing these two cases, we confirm there is no immersion effect when the focal spot is projected through the planar interface. Since the depth of the planar layer is only $200 \mathrm{~nm}$, spherical aberration is negligible, and there is practically no degradation of the focus in Fig. 4(b) compared to the case of Fig. 4(a). Third, we focus on the nano-SIL. A comparatively smaller focal spot is observed, as shown in Fig. 4(c).

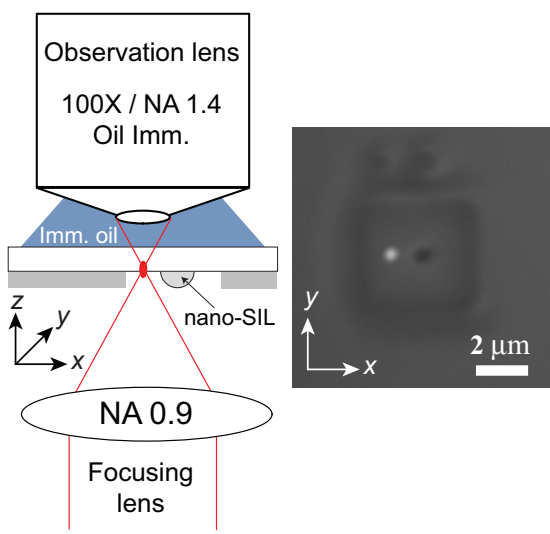

(a)

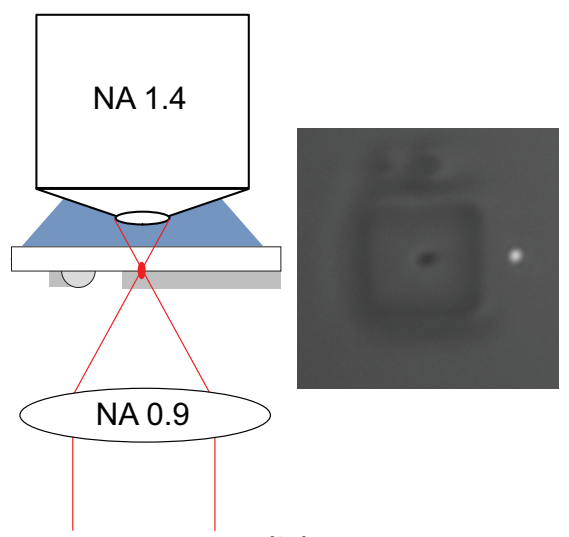

(b)

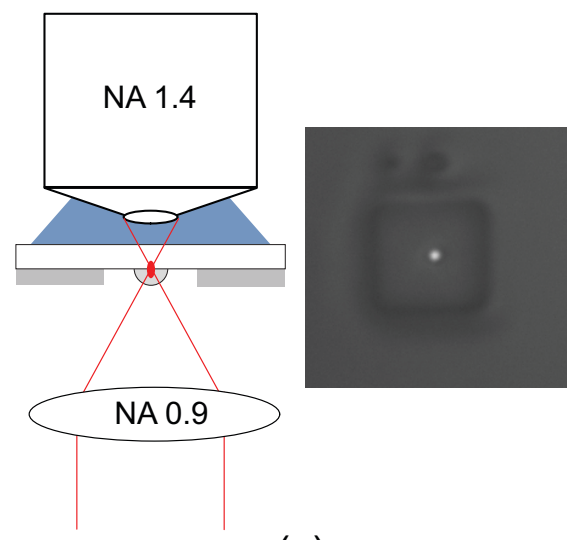

(c)

Figure 4. Geometries of the optical characterization experiments and the corresponding CCD images $(x-y$ plane): focal spot (a) at the interface between air and glass in an area without polymer; (b) at the glass-polymer interface illuminated through a 200-nm thick planar polymer; and (c) illuminating the 700-nm nano-SIL. 
We evaluate the reduction of the spot size by comparing the amplitude and phase distributions of the focal spots from the cases of Figs. 4(a) and 4(c) for both 700-nm and 530-nm SILs. The transverse and longitudinal cross-sections of the 3D measurement data through the peak intensity points are shown in Fig. 5. For the Airy disc size, the transverse $(x-y)$ phase maps are of significant interest since the central iso-phase disc represents the Airy disc.

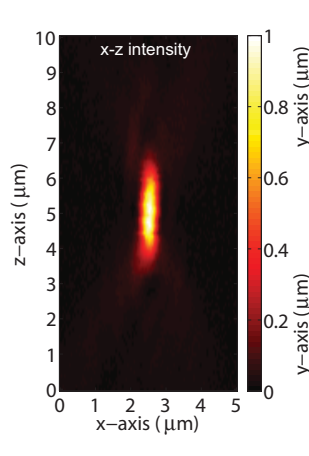

(a)

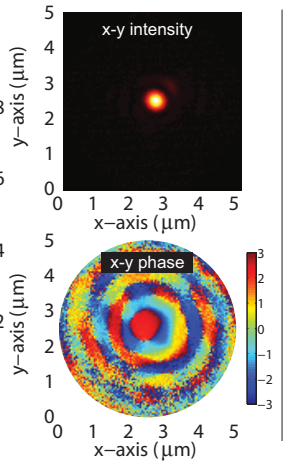

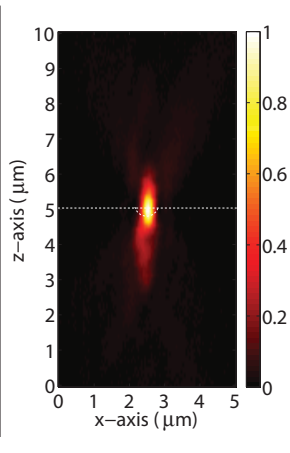

(b)

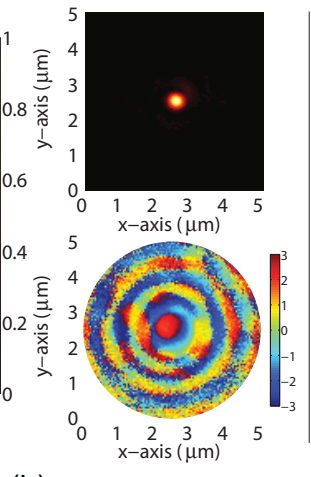

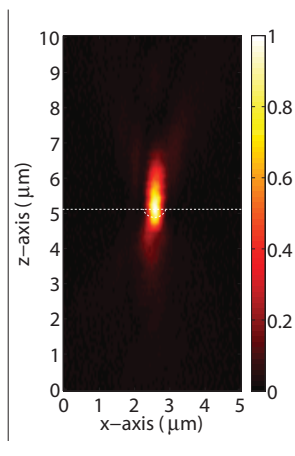

(c)

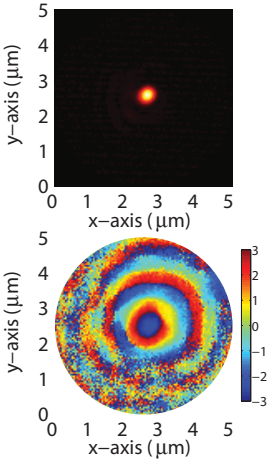

Figure 5. (Color online) Cross-sections of the measured 3D fields through the maximum intensity point. (a) The focal spot at the interface between air and glass substrate shown in Fig. 4(a). (b) The focal spot on the 700-nm SIL shown in Fig. 4(c). (c) The focal spot on the 530-nm SIL. The intensities are normalized and the position of the SIL is indicated in the longitudinal $(x-z)$ intensity distributions.

The high-resolution interference microscope produces both amplitude and phase data for the observed optical fields, and therefore can provide super-resolution, in other words, exceed the diffraction limit. In particular, the lateral position accuracy of specific phase features, such as the position of phase jumps or singularities, are not subject to the diffraction $\operatorname{limit}^{23}$. For example, since singularities are mathematically exact points, measuring their distance or position is only limited by the signal-to-noise ratio, and therefore super-resolution is generally possible. However, this super-resolution does not refer to a physical object but only to particular topological features in the wave-field. Therefore, the resolution in the $x-y$ phase plane is in general given by the demagnified size of the pixels from the image (camera) plane to the object plane. The HRIM demonstrated a lateral position accuracy down to $20 \mathrm{~nm}$ for wave-fields in our previous work ${ }^{24}$; the current setup provides a $100-\mathrm{nm}$ accuracy. In this way, the phase fields provide a more precise measure of the spot size reduction. For the transverse (x-y) phase measurements at the focal plane, the comparison of the size of the central iso-phase discs, which represent the Airy disc of the intensity distributions (1.22 $\lambda / \mathrm{NA})$, directly shows the reduction of the full spot size rather than the FWHM spot size (0.5 /NA) in the amplitude measurements.

The NA0.9 focusing objective leads to a predicted Airy disc size of $870 \mathrm{~nm}$ and FWHM spot size of $360 \mathrm{~nm}$ at $642 \mathrm{~nm}$ wavelength. For the reference case [see Fig. 4(a)], the Airy disc size is measured to be $880 \mathrm{~nm}$ and the FWHM spot size is measured to be $400 \mathrm{~nm}$, as shown in Fig. 5(a). These results show good agreement with the expected values. The results shown in Fig. 5(b) allow the measurement of the Airy disc (full spot) and the FWHM spot sizes for the 700-nm nano-SIL, which are found to be $650 \mathrm{~nm}$ and $336 \mathrm{~nm}$, respectively. The equivalent results for the $530 \mathrm{~nm}$ nano-SIL are shown in Fig. 5(c), and yield results of $660 \mathrm{~nm}$ for the full spot size and $337 \mathrm{~nm}$ for the FWHM. As discussed above, the super-resolution performance due to the amplitude and phase measurement data provided by the HRIM, allows evaluation of the spot size reduction of the full spot with an accuracy of $100 \mathrm{~nm}$ in the current setup. The measured reduction ratio, i.e. the reference spot size divided by the nano-SIL spot size, is approximately 1.35 . We assume that the subhemispherical shape ${ }^{12}$ of the nano-SIL is the cause of the lower reduction ratio than would be expected given the refractive index of the nano-SIL $(\mathrm{n}=\sim 1.5)$.

We examine the intensity enhancement, which is caused by the immersion effect, by comparing the lateral and axial intensity profiles of the reference focal spot and the immersion focal spot for the 700-nm SIL as shown in Fig. 6. The peak intensity is enhanced approximately $50 \%$ by the immersion effect due to stronger spatial confinement of the field. The power of the Gaussian beam is given as

$$
P=\frac{1}{2} \pi w^{2} I,
$$


where $I$ is the peak intensity and $w$ is the beam waist ${ }^{25}$. From Eq. (7), when there is no absorption and loss $[P=$ constant $]$ the peak intensity value is proportional to the square of the spot size. The spot size reduction of 1.35 leads to the peak intensity enhancement of $1.82\left(=1.35^{2}\right)$. As the measured value is 1.48 , there is discrepancy mainly due to the size of the SIL $(=700 \mathrm{~nm})$, which is smaller than the full focal spot (the Airy disc $=880 \mathrm{~nm})$. This result shows a qualitative increase in the peak intensity due to the immersion effect, and we expect that a nano-SIL with a shape closer to the ideal hemispherical shape would exhibit characteristics closer to the predicted value.

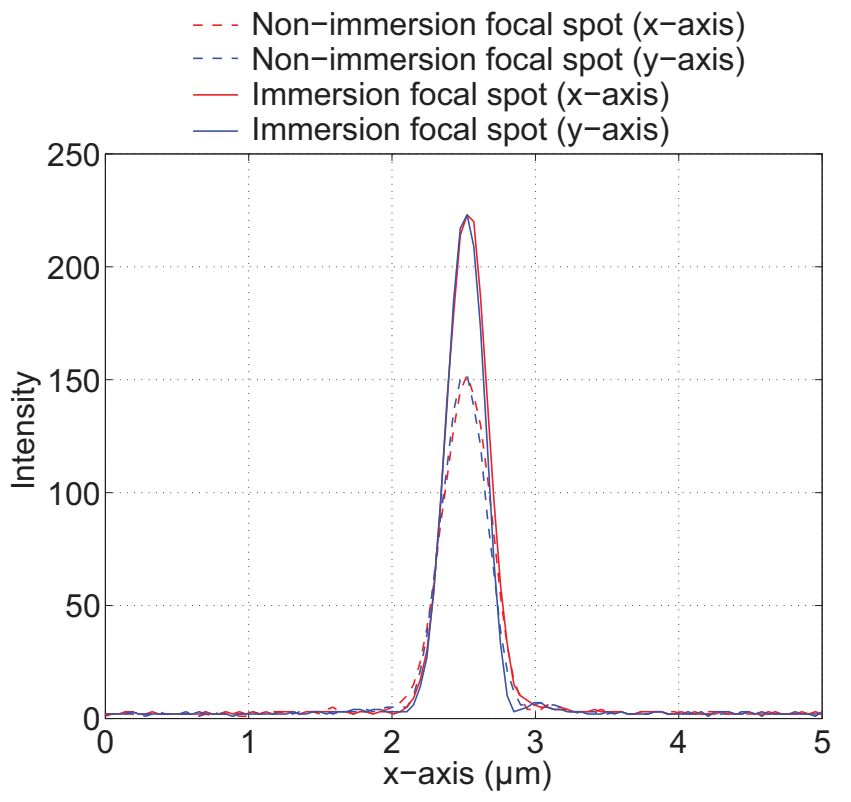

(a)

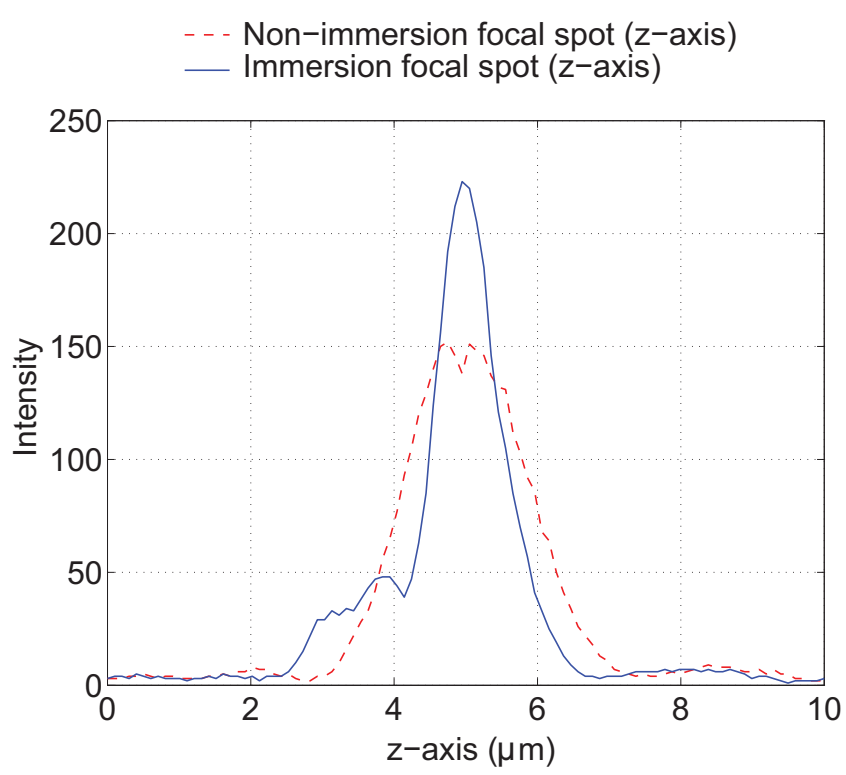

(b)

Figure 6. (Color online) Lateral ( $x$ - and $y$-axes) and axial (z-axis) intensity profiles of the reference and 700-nm SIL focal spots: (a) $x$ - and $y$-axes and (b) $z$-axis profiles. Dashed lines denote the reference focal spot and solid lines denote the focal spot of the 700-nm nano-SIL.

\section{CONCLUSION}

We have experimentally realized for the first time nanometer-scale SILs down to subwavelength size. The details of the fabrication and characterization of these devices are persented. The nano-SILs were fabricated by EBL and thermal reflow of the PMMA structures, and these reflowed structures are replicated on glass substrates by soft lighotraphy for optical characterization. Using the HRIM, we measured the 3D amplitude and phase distributions to show the reduction of the FWHM spot and the full spot (Airy disc) sizes, respectively. The reduction ratio for the full spot size was found to be 1.35 rather than the refractive index of the SIL of 1.5. The subhemispherical shape is assumed to be the cause of the lower reduction ratio. The enhancement of the peak intensity provides an additional confirmation of the immersion effect, i.e. tighter focusing due to the nano-SIL. Prospective applications of such nano-SILs include near-field focusing and imaging $^{12}$, optical data storage ${ }^{26}$, and maskless direct-write lithography ${ }^{27}$. Furthermore, the introduction of plasmonic structures on the bottom of the SIL could further improve performance and resolution ${ }^{28}$.

\section{ACKNOWLEDGMENTS}

The research leading to these results has received funding from the European Community's Seventh Framework Programme FP7-ICT-2007-2 under grant agreement No. 224226.

\section{REFERENCES}

[1] Hooke, R., [Lectures and Collections; Microscopium], Royal Society, London, 1 (1678).

[2] Brocksch, D., [Innovation] The Magazine form Carl Zeiss, vol. 15, 8 (2005). 
[3] Abbe, E., "On New Methods for Improving Spherical Correction applied to the Construction of Wide-angled Object-glasses," J. of the Royal Microscopical Society 2, 812-824 (1879).

[4] Abbe, E., "On the Estimation of Aperture in the Microscope," J. of the Royal Microscopical Society 2 1, 388-423 (1881).

[5] Mansfield, S. M. and Kino, G. S., "Solid immersion microscope," Appl. Phys. Lett. 57, 2615-2616 (1990).

[6] Brunner, R., Burkhardt, M., Pesch, A., Sandfuchs, O., Ferstl, M., Hohng, S. and White, J. O., "Diffraction-based solid immersion lens," J. Opt. Soc. Am. A 21, 1186-1191 (2004).

[7] Fletcher, D. A., Crozier, K. B., Guarini, K. W., Minne, S. C., Kino, G. S., Quate, C. F. and Goodson, K. E., "Microfabricated silicon solid immersion lens," JMEMS, vol. 10, pp. 450-459 (2001).

[8] Lang, M., Milster, T. D., Aspnes, E., Minamitani, T. and Borek, G., "Investigation of micro solid immersion lens mounting systems," Jpn. J. Appl. Phys. 46, 3737-3740 (2007).

[9] Kishia, T., Shibata, S. and Yano, T., "Fabrication of high-refractive-index glass micron-sized solid immersion lenses by a surface-tension mold technique," J. Non-Crystalline Solids 354, 558-563 (2008).

[10] Brun, M., Richard, M. and Nicoletti, S., "Integrated Micro Solid Immersion Lens for Near Field Optical Data Storage," International Symposium on optical memory (ISOM09), Mo-E-04 (2009).

[11] Lee, J. Y., Hong, B. H., Kim, W. Y., Min, S. K., Kim, Y., Jouravlev, M. V., Bose, R., Kim, K. S., Hwang, I. C., Kaufman, L. J., Wong, C. W., Kim, P. and Kim, K. S., "Near-field focusing and magnification through selfassembled nanoscale spherical lenses," Nature 460, 498-501 (2009).

[12] Mason, D. R., Jouravlev, M .V. and Kim, K. S., "Enhanced resolution beyond the Abbe diffraction limit with wavelength-scale solid immersion lenses," Opt. Lett. 35, 2007-2009 (2010).

[13] Kim, M.-S., Scharf, T., Haq, M. T., Nakagawa, W. and Herzig, H. P., "Subwavelength-size solid immersion lens," Opt. Lett. 36, 3930-3932 (2011).

[14] Nussbaum, Ph., Völkel, R., Herzig, H. P., Eisner, M. and Haselbeck, S., "Design, fabrication and testing of microlens arrays for sensors and microsystems," Pure Appl. Opt. 6, 617-636 (1997).

[15] Ottevaere, H., Cox, R., Herzig, H. P., Miyashita, T., Naessens, K., Taghizadeh, M., Völkel, R., Woo, H. J. and Thienpont, H., "Comparing glass and plastic refractive microlenses fabricated with different technologies," J. Opt. A: Pure Appl. Opt. 8, S407-S429 (2006).

[16] Schleunitz, A. and Schift, H., "Fabrication of 3D nanoimprint stamps with continuous reliefs using dose-modulated electron beam lithography and thermal reflow," J. Micromech. Microeng. 20, 095002 (2010).

[17] Lee, H.-M., Chang, E. Y., Chen, S.-H. and Chang, C.-Y., "New nanometer T-gate fabricated by thermally reflowed resist technique," Jpn. J. Appl. Phys. 41, 1508-1510 (2002).

[18] Grossmann, T., Hauser, M., Beck, T., Gohn-Kreuz, C., Karl, M., Kalt, H., Vannahme, C. and Mappes, T., "High-Q conical polymeric microcavities," Appl. Phys. Lett. 96, 013303 (2010).

[19] Xia, Y. and Whitesides, G. M., "Soft Lithography," Annu. Rev. Mater. Sci. 28, 153-184 (1998).

[20] Kim, M.-S., Scharf, T. and Herzig, H. P., "Small size microlenes characterization by Multiwavelength High Resolution Interference Microscopy," Opt. Express 18, 14319-14329 (2010).

[21] Kim, M.-S., Scharf, T., Mühlig, S., Rockstuhl, C. and Herzig, H. P., "Engineering photonic nanojets,” Opt. Express 19, 10206-10220 (2011).

[22] Kim, M.-S., Scharf, T., Mühlig, S., Rockstuhl, C. and Herzig, H. P., "Gouy Phase Anomaly in Photonic Nanojets," Appl. Phys. Lett. 98, 191114 (2011).

[23] Rockstuhl, C., Märki, I., Scharf, T., Salt, M., Herzig, H. P. and Dändliker, R., "High Resolution Interference Microscopy: A Tool for Probing Optical Waves in the Far-Field on a Nanometric Length Scale," Current Nanoscience 2, 337-350 (2006).

[24] Rockstuhl, C., Salt, M. and Herzig, H. P., "Theoretical and experimental investigation of phase singularities generated by optical micro- and nano-structures,” J. Opt. A: Pure Appl. Opt. 6, 271-276 (2004).

[25] Siegman, A. E., [Lasers], University Science books, 665 (1986).

[26] Terris, B. D., Mamin, H. J. and Ruger, D., "Near-field optical data storage using a solid immersion lens," Appl. Phys. Lett. 65, 388-390 (1994).

[27] Ghislaina, L. P., Elings, V. B., Crozier, K. B., Manalis, S. R., Minne, S. C., Wilder, K., Kino, G. S. and Quateb, C. F., "Near-field photolithography with a solid immersion lens," Appl. Phys. Lett. 74, 501-503 (1999).

[28] Kim, Y., Kim, S., Jung, H., Lee, E. and Hahn, J. W., "Plasmonic nano lithography with a high scan speed contact probe," Opt. Express 17, 19476-19485 (2009). 